\title{
Review
}

\section{Exercise and the Aging Brain: Considerations for Sex Differences}

\author{
Cindy K. Barha ${ }^{\mathrm{a}, \mathrm{b}}$ and Teresa Liu-Ambrose ${ }^{\mathrm{a}, \mathrm{b}, \mathrm{c}, *}$ \\ ${ }^{a}$ Djavad Mowafaghian Centre for Brain Health, Vancouver, Canada \\ ${ }^{\mathrm{b}}$ Department of Physical Therapy, University of British Columbia, Vancouver, Canada \\ ${ }^{\mathrm{c}}$ Centre for Hip Health and Mobility, Vancouver, Canada
}

Accepted: 28 July 2018

Published: 20 August 2018

\begin{abstract}
Engaging in targeted exercise interventions is a promising, non-pharmacological strategy to mitigate the deleterious effects of aging and disease on brain health. However, despite its therapeutic potential, a large amount of variation exists in exercise efficacy in older adults aged 55 and older. In this review, we present the argument that biological sex may be an important moderator of the relationship between physical activity and cognition. Sex differences exist in dementia as well as in several associated risk factors, including genetics, cardiovascular factors, inflammation, hormones and social and psychological factors. Different exercise interventions, such as aerobic training and resistance training, influence cognition and brain health in older adults and these effects may be sex-dependent. The biological mechanisms underlying the beneficial effects of exercise on the brain may be different in males and females. Specifically, we examine sex differences in neuroplasticity, neurotrophic factors and physiological effects of exercise to highlight the possible mediators of sex differences in exercise efficacy on cognition. Future studies should address the potential sex difference in exercise efficacy if we are to develop effective, evidence-based exercise interventions to promote healthy brain aging for all individuals.
\end{abstract}

Keywords: Exercise, sex differences, brain, cognition, aging, dementia, Alzheimer's disease, neuroplasticity, neurotrophic factors, physical activity

\section{INTRODUCTION}

Worldwide, over 47 million people suffer from dementia and this number is expected to reach 74.7 million by the year 2030 and 131.5 million by 2050 ; the estimated total worldwide economic burden will be 2 trillion dollars by 2030 [1]. In the face of these staggering numbers, the societal value of identifying and developing effective intervention and prevention

\footnotetext{
*Correspondence to: Dr. Teresa Liu-Ambrose, Aging, Mobility, and Cognitive Neuroscience Lab, Djavad Mowafaghian Centre for Brain Health, c/o Liu-Ambrose Lab, 2215 Wesbrook Mall, Vancouver BC, V6T 2B5, Canada. Tel.: +1 604 875 4111/Ext. 69056; E-mail: teresa.ambrose@ubc.ca.
}

strategies is of utmost importance [2]. Effective pharmacological treatments of dementia remain elusive. Engaging in targeted exercise interventions is a promising, non-pharmacological strategy to mitigate the deleterious effects of aging and disease on brain health [3, 4]. However, despites its therapeutic potential, a large amount of variation exists in exercise efficacy [5-8]. To maximize effectiveness, it is vital to understand the sources of this variation and to identify factors that increase the likelihood of positive cognitive outcomes from exercise interventions. Given the greater prevalence of $\mathrm{AD}$ and faster rate of progression from mild cognitive impairment (MCI) to $\mathrm{AD}$ in females compared with males [9], 
there is a need to assess potential sex differences in treatment efficacy, including different exercise interventions. Unless otherwise specifically stated, this review focuses on older adults, aged 55 and older.

\section{SEX DIFFERENCES IN DEMENTIA AND ASSOCIATED RISK FACTORS}

Sex differences exist in dementia, although the relationships are complex and multifactorial. Females are disproportionally affected by AD, showing twofold greater risk at later ages [10], faster progression of brain atrophy (1-2.5\% per year) [11, 12] and greater AD-related pathology [13-15] than men. Conversely, some studies find that males are at higher risk for MCI, a prodromal stage between normal age-appropriate cognitive changes and dementia [16], for both the amnestic and non-amnestic type [17-19]; although, not all studies find this maleadvantage [20, 21]. Recently, it has been suggested that diagnosis of MCI may be delayed in females, which may be responsible for the possible increased risk for MCI seen in males. Specifically, females show greater verbal memory performance than males, despite similar levels of neurodegeneration [22, 23]. This finding suggests that females have cognitive reserve within this domain which delays manifestation of deficits until greater levels of pathology are present.

\section{Sex differences in genetic risk factors}

Sex differences also exist in risk factors for dementia. Apolipoprotein E $\varepsilon 4$ (APOE4) is the greatest genetic risk factor for AD [24], and despite similar frequencies, females show greater detrimental effects. Female carriers of the $\varepsilon 4$ allele have about a 1.5x higher risk for AD [25-28], more amyloid plaques and neurofibrillary tangles [29] and present with greater verbal memory declines over time [30] compared with male carriers. Further, APOE4 carriers show lower serum levels of BDNF, an association only seen in females with AD [31]. On the other hand, levels of cerebral microbleeds, a component of vascular cognitive impairment, are exacerbated (2fold) in APOE4 males compared with females [32]. Further, recent work suggests the greater risk associated with carrying one copy of the $\varepsilon 4$ allele in females may only be seen at younger ages, specifically between 65 and 75 years [26]. Thus the relationship between APOE4, sex and dementia is not straightforward.

\section{Sex differences in cardiovascular risk factors}

Peripheral cardiovascular risk factors for dementia include hypertension, type 2 diabetes, obesity, atherosclerosis, and hypercholesterolemia. Cardiovascular risk profiles vary by sex and thus, their contributions to the development of dementias could also differ by sex [33]. Indeed, evidence does suggest the contribution of some cardiovascular risk factors may be greater in older females than older males $[34,35]$. For example, the increased risk for vascular cognitive impairment from type 2 diabetes is $19 \%$ higher in older females than males [36]. In males, total cholesterol, a biomarker of cardiovascular health, is strongly associated with AD symptoms [37]. Further, a sex difference exists in vascular aging, a main risk factor for cardiovascular disease. Specifically, endothelial dysfunction, a key component of vascular aging, occurs gradually in males commencing approximately in midlife (approximately the third decade of life in this study), whereas in females, commencement is delayed and accelerates after the onset of menopause and loss of estrogens [38].

\section{Sex differences in inflammation as a risk factor}

Systemic and central (neuro) inflammation is a risk factor for $\mathrm{AD}$ and other dementias, and may be a stronger risk for aged females [37]. Aging is accompanied by a chronic, low-grade inflammatory phenotype evidenced by elevated serum levels of several pro-inflammatory cytokines including IL-6 and TNF- $\alpha$ and decreased levels of antiinflammatory cytokines including IL-10 [39]. High levels of pro-inflammatory cytokines are related with cognitive impairment, MCI and AD [40]. Importantly, in humans the association between increased peripheral levels of pro-inflammatory cytokines and cognitive impairment is seen in older females but not older males [39, 41]. Within the brain, agerelated induction of neuro-inflammatory genes differ between males and females, with females showing enhanced expression [42, 43]. Likewise, the aged female hippocampus presents with $25-40 \%$ more activated microglia, the brain's resident immune cells that secrete cytokines, and astrocytes compared to age-matched males [44]. Interestingly, accelerated or increased microglial development has been seen in AD brains compared to age-matched controls, and in mice, females show faster microglial maturation in the hippocampus than males [45], suggesting that microglia may be involved in the 
sex differences seen in AD. Taken together, this suggests that sex differences may exist in the association among inflammation and dementia. However, whether inflammation is involved in the etiology or progression of dementia or both is currently under investigation [46].

\section{Sex differences in hormones as a risk factor}

Sex-specific risk factors for cognitive impairment also include loss of sex steroid hormones with age. In women, estrogens are rapidly lost with the onset of menopause which occurs on average at 51 years of age, whereas in men the loss of bioavailable testosterone is much more gradual with approximately $1-2 \%$ reduction per year beginning in the third decade of life [47, 48]. Menopause is associated with increased levels of $\mathrm{AD}$ biomarkers including hypometabolism, $\mathrm{A} \beta$ deposition, and decreased hippocampal volume [49] and surgical menopause that occurs prior to natural menopause is further associated with an increased risk of $\mathrm{AD}$ [50]. Use of hormone replacement therapy (HRT) may reduce this risk [51]. However, timing of HRT commencement in relation to estrogen loss is important as initiation of HRT years after menopause is associated with increased risk of dementia, whereas initiation closer to the onset of menopause may not be [52]. Furthermore, the type of estrogen taken is of considerable importance, as estrone and estradiol have different effects on cognitive performance [53-55].

\section{Sex differences in social and psychological risk} factors

Social and psychological risk factors for dementia may also differ by sex. For example, higher education and occupational attainment are protective factors in both females and men, as they are believed to increase cognitive reserve delaying the onset of cognitive deficits through engagement of compensatory mechanisms [56-59]. However, historically, females have had less opportunities to obtain higher education than men, thus low education may be a greater risk factor for females [60]. Women are most often the primary caregivers for people with dementia [61], a source of considerable psychological stress with physiological consequences. For example, premenopausal female caregivers (average age 38 ) show more rapid cellular aging, as indexed by telomere length [62]. Interestingly, although spousal caregivers of both sexes show significantly increased risk of developing dementia, the risk is $3 \mathrm{x}$ higher in male caregivers [63].

\section{EFFECTS OF DIFFERENT EXERCISE INTERVENTIONS OF COGNITION AND BRAIN IN OLDER ADULTS}

Broadly, there are two distinct forms of exercise: 1) aerobic training (AT; e.g., running, walking), aimed at improving cardiovascular health; 2) resistance training (RT; e.g., lifting weights), aimed at improving muscle strength. Although most research to date has focused on AT, both forms of exercise have beneficial effects on cognitive and brain plasticity in older adults [3, 64-67], although the underlying mechanisms may be different. Longitudinal cohort studies that rarely differentiate between types of exercise, show engaging in physical activity is associated with less cognitive decline over time in older adults [68-71]. Results from randomized controlled trials (RCTs) of targeted interventions provide stronger support for the relationship between exercise and cognitive functioning.

Meta-analyses of RCTs in older adults show that engaging in targeted AT programs promotes cognitive performance $[5,8,67,72,73]$, although others have found modest to minimal or no effects [6, 74-76]. The beneficial effect of AT on cognitive functioning has been seen across different clinical and non-clinical populations, including MCI, vascular dementia and cognitively healthy older adults [77-80]. Studies suggest executive functions are the cognitive domain that most benefits from AT $[5,81]$. Moreover, AT interventions can lead to changes in brain structure, activation, and connectivity, indicating enhanced functional brain plasticity [for example see 79, 82, 83-87], and recent work suggest that brains that are organized with greater connections within modules and less connections between modules are more likely to show AT-dependent gains in executive functions [88].

Although much less studied, RT in older adults is also beneficial for cognitive functioning [8, 67, 75]. In one of the first large-scale RCTs of RT, LiuAmbrose and colleagues showed that engaging in progressive RT at 2 doses, once or twice per week, for 12 months improved the executive functions of selective attention and conflict resolution in older women [89], effects that were maintained for an additional 12 months post-training [90]. Previous to this RCT, evi- 
dence from 2 smaller trials in men only indicated that RT was beneficial for cognition [91, 92]. Associative memory performance is also enhanced with 6 months of RT in older women [93]. The cognitive-gains from RT are further reflected in hemodynamic effects within the brain. Specifically, RT in older women led to functional changes within regions involved in executive functions - the anterior portion of the left middle temporal gyrus and the left anterior insula extending into the lateral orbital frontal cortex [94]; and associative memory - right lingual, occipitalfusiform, and right frontal pole [93]. Furthermore, twice-weekly RT reduced volume of white matter lesions [95] and reduced cortical white matter atrophy [90].

Despite common cognitive outcomes, AT and RT exert their benefits through distinct physiology and are possibly subserved by both divergent and common mechanistic pathways. For example, AT increases cardiovascular fitness as measured by maximum oxygen uptake while RT increases muscle mass and strength [96]. Work in young adult male rats suggests that while both AT and RT improved spatial learning and memory, AT preferentially increased brain-derived neurotrophic factor (BDNF) while RT preferentially increased a different neurotrophic factor, insulin-like growth factor-1 (IGF-1) [97]. On the other hand, both types of training reduce cardiometabolic risk factors for neurodegeneration [89, 98-103] and associated systemic inflammation [64, 96, 104, 105] to different degrees. Studies are required to compare and contrast these two forms of exercise as well as examine their combined influence on cognition and brain function.

\section{POSSIBLE SEX DIFFERENCES IN EXERCISE EFFICACY}

\section{Possible sex differences in exercise efficacy: Meta-analytic findings}

Older females greater than 65 years of age are more sedentary and engage in less physical activity than age-matched males [106, 107] and being sedentary may have greater negative impact on processing speed in older age in females then males [108]. Thus increasing physical activity levels in females may have a greater impact on cognition. Colcombe and Kramer [5] first suggested that females greater than 55 years of age may show greater cognitive benefits from AT. This was recently confirmed in a meta-analysis of RCTs showing that sex moderates the effect of exercise on cognitive function [8]. Specifically, AT was associated with a larger effect size in studies that utilized a higher percentage of females (over 71\%) than studies with lower percentage of females for executive functions (effect sizes: 2.83 vs. 1.46) [8]. A similar female-advantage was found for rodent studies that utilized forced AT (effect size for female studies: 1.24; effect size for male studies: 0.53) but not voluntary AT for hippocampus-dependent learning and memory [109]. Further, human studies of RT as well as multimodal training (e.g., combined AT and RT) also show the female-advantage for executive functions [8].

\section{Possible sex differences in exercise efficacy: $R C T$ findings}

Direct support for the supposition that older females show greater cognitive benefits from exercise than males is provided by a recent secondary analysis of a RCT of 6 months, 3 times per week progressive AT in participants with mild subcortical ischemic vascular cognitive impairment. Specifically, a significant interaction was found between treatment group (AT or control) and biological sex, with AT significantly improving the executive function of set-shifting in females (36\% improvement in performance from baseline) but not males ( $31 \%$ decline in performance from baseline), an effect that was retained 6 months after trial termination [110]. Additional support for a possible sex difference in AT efficacy is found in two RCTs that stratified analyses by sex. In participants with MCI, Baker et al., [77] found AT increased performance compared to controls on 3 cognitive tests in females but only on 1 test in males. Additionally, increased adherence to a 12 month AT program was associated with improved attention and memory in older females with MCI and with only memory in older males [111]. Although no RCT has examined sex differences in brain outcomes, in a cross-sectional study Varma et al., [112] found that greater amounts of objectively measured walking activity over one week were significantly associated with larger hippocampal volumes among older females but not among males and, in another study, they found greater enhancements in the volume of the subiculum, which is part of the posterior hippocampus in females only [113]. Together these findings indicate that engaging in AT leads to greater beneficial effects on cognition in females than males. 


\section{HOW CAN SEX MODERATE EXERCISE EFFICACY?}

Despite the many studies investigating the impact of exercise on cognitive and brain function in humans, comparatively little is known about the biological mechanisms underlying these effects, and whether these vary by sex is yet to be examined. Our current understanding of how exercise promotes cognitive and brain function largely stems from animal studies and is mainly restricted to AT as there is a dearth of mechanistic evidence for RT. Cotman [64] proposed an integrative model by which AT enhances cognition, brain function, and neuroplasticity through induction of neurotrophic factor cascades (i.e., BDNF, IGF-1, vascular endothelial growth factor (VEGF)). In support of this model, rodent studies indicate that central BDNF levels mediate the beneficial effects of AT on the brain $[4,65,66,114$, 115]. In humans, although AT-induced increases in peripheral levels of BDNF are generally seen, some studies do fail to find this effect [116-119] and biological sex may be an important moderating factor [116]. BDNF supports neuronal survival and growth, synaptic plasticity, is involved in cellular mechanisms required for learning and memory, and sex differences exist in some of its functions [120-124]. Furthermore, AT benefits cognition through enhancements in neuroplasticity, including hippocampal neurogenesis; processes which also show sex differences. Physiological responses to exercise may also underlie the beneficial effects on the brain, and these may also help explain the sex difference in AT efficacy on cognition.

\section{Sex differences in neuroplasticity}

Rodent studies show that running benefits cognition through alterations in neuroplastic processes in key brain regions involved in learning and memory, including the hippocampus and prefrontal cortex (PFC) [for review see 4, 65, 66, 125]. Neuroplasticity, the ability of the adult brain to change, remodel and reorganize in response to the environment, includes changes in dendritic branching, synaptogenesis, angiogenesis, and neurogenesis. Many of these forms of neuroplasticity show sex differences and are altered by exposure to sex hormones, such as estradiol and testosterone [55, 126, 127]. Although a complete review of this topic is beyond the scope of this manuscript, we include a few examples to provide evidence that sex differences in exercise efficacy may be related to sex differences in neuroplasticity.

Sex differences exist in neuroplastic mechanisms at the structural, cellular, and molecular levels. Dendritic spine density on neurons in the CA1 region of the hippocampus respond to estradiol in female but not male rodents [128, 129], whereas spine density responds to testosterone in both females and males $[129,130]$. Further, chronic stress causes retraction of apical dendrites of CA3 neurons of the hippocampus [131] and shrinkage of certain dendrites in the medial PFC [132] in males but not female rats. Adult hippocampal neurogenesis, the production of new neurons in the dentate gyrus in adulthood, is involved in learning and memory. Although female and male rodents do not seem to differ in overall basal levels of hippocampal neurogenesis, sex differences do emerge in response to stimulation including hormones and behavior [55, 127, 133]. For example, adult male rats show increases in hippocampal neurogenesis in response to spatial water maze training, a task with a male-advantage in performance, but females do not [134]. On the other hand, compared to males, females show greater increases in hippocampal neurogenesis after training on a task with a female-advantage in performance [135]. Further, exposure to stress in adolescence leads to sex-specific effects on hippocampal neurogenesis in adulthood, with female rats showing decreased and male rats showing increased levels of new neurons [136]. Another example of sex differences in neuroplasticity is seen in the response to chronic estradiol treatment of hippocampal neurogenesis, which was seen only in female rats and not male rats [137]. Sex differences extend to the molecular level, as signalling pathways subserving synaptic plasticity also differ between the sexes, including the calcium/calmodulin kinase kinase (CaMKK) pathway [for review see 126].

\section{Sex differences in $B D N F$}

BDNF is vital for neuronal health, survival and plasticity and, importantly, sex differences exist in its functioning [120-124]. The promoter region of the BDNF gene contains an estradiol-response-like element [138] and BDNF protein levels fluctuate across the estrus (in rodents) and menstrual (in humans) cycles with increases in estradiol associated with greater BDNF expression [139]. Within the mossy fiber pathway of the hippocampus BDNF expression is sex-dependent, with estradiol upregulating and testosterone suppressing BDNF levels [140]. Interest- 
ingly, BDNF levels decline with age and functionally, this decline is related to impaired cognitive function in older females but not males [141]. Further evidence of sex differences in BDNF function are seen in studies of the BDNF Val66Met polymorphism; the Met allele is related to reduced activity-dependent secretion of the mature form of BDNF from neurons [142]. There are sex differences in the effects of the Met allele on hippocampal blood flow, age-related cognitive and brain volume decline and on AD risk [143-146]. Further, in the BDNF Val66Met mouse model, estradiol interacts with the Met allele to influence hippocampal memory and hippocampal BDNF expression across the estrous cycle [147]. Although not directly examined yet, there is some suggestion that sex differences exist in the AT-induced increase in circulating BDNF. In a meta-analysis of 9 studies, AT effects on BDNF levels were greater in studies utilizing female rodents compared to studies with male rodents (effect size for female studies: 2.59; effect size for male studies: 0.46) [109]. Furthermore, in humans, 6 months of AT increased circulating BDNF levels to a greater extent in females than males [110]. Therefore, sex differences in BDNF functioning may extend to AT-induction of BDNF.

\section{Sex differences in the physiological adaptations to exercise}

Sex differences exist in several key systems involved in exercise - including the respiratory system, musculoskeletal system, and cardiovascular system - as well as in the physiological responses of these systems to exercise training. Inherent functional and anatomical differences exist between the sexes in lung size and volume, airway diameter, diffusion surface, and maximal expiratory flow rates that affect exercise capacity across the lifespan, with females at a disadvantage [148, 149]. Age-dependent declines in aerobic capacity as measured by maximal oxygen uptake (V02 max) are greater in males than females in later life (60 years and older) [150, 151]. Premenopausal females and age-matched males differ in whole-body fuel use with females utilizing more lipids and males utilizing more carbohydrates and protein, which is related to sex differences in expression of genes associated with fat and carbohydrate metabolism and the sex hormone estradiol [152]. Muscle mass, strength and quality decline with age [153], and strength exercises help maintain and improve these indices in older adults [154]; how- ever, the degree of benefit may differ by sex with males showing greater effects [155], although this is not consistently found [156]. Interestingly, supplementation with fish-oil in conjunction with RT was more effective in improving muscle quality in older females than males [157]. Age-associated changes in the cardiovascular system differ between the sexes [158] and influence exercise capacity. For example, decreases in maximal cardiac power output and reserve and maximal oxygen uptake seen in older males are preserved in older females [150, 159]. Conversely, studies report greater beneficial effect of exercise on some hemodynamic responses in older males than females, including lower blood pressure [160] and increased leg blood flow [161] in response to AT. Interestingly, these differences between males and females in the physiological responses to exercise may be related to sex differences in effective dose of exercise [for review see 161] and future studies are required to examine this. Further, engaging in chronic exercise attenuates age-associated endothelial dysfunction in older males [162, 163]. However, in older females the beneficial effects of exercise on endothelial function are diminished $[162,163]$ and may be dependent on the presence of estrogens [164].

\section{FUTURE STUDIES AND CONCLUDING REMARKS}

Engaging in physical activity is a promising, nonpharmacological strategy to mitigate the deleterious effects of aging and disease on cognitive and brain health. However, to maximize the utility of exercise, interventions should be targeted and tailored to specific populations and move beyond the 'onesize-fits-all' approach. Specifically, we argue that biological sex may be an important factor that moderates the relationship between exercise and cognition. A large gap exists in the current knowledge as few studies of exercise and brain health have directly examined this potential sex difference. It is also currently not known whether the proposed mechanisms underlying exercise effects on the brain differ between the two sexes. Thus future studies of RT and AT as well as alternative forms of exercise such as yoga, should address the potential sex difference in exercise efficacy if we are to develop effective, evidence-based exercise interventions to promote healthy brain aging for all individuals. 


\section{REFERENCES}

[1] Prince M, et al. World Alzheimer Report 2015. 2015, Alzheimer's Disease International (ADI): London.

[2] Brookmeyer R, et al. Forecasting the global burden of Alzheimer's disease. Alzheimers Dement. 2007;3(3):18691.

[3] Bherer L, Erickson KI, Liu-Ambrose T. A review of the effects of physical activity and exercise on cognitive and brain functions in older adults. J Aging Res. 2013;2013:657508.

[4] Duzel E, van Praag H, Sendtner M. Can physical exercise in old age improve memory and hippocampal function? Brain. 2016;139(Pt 3):662-73.

[5] Colcombe S, Kramer AF. Fitness effects on the cognitive function of older adults: A meta-analytic study. Psychol Sci. 2003;14(2):125-30.

[6] Gates N, et al. The effect of exercise training on cognitive function in older adults with mild cognitive impairment: A meta-analysis of randomized controlled trials. Am J Geriatr Psychiatry. 2013;21(11):1086-97.

[7] Ohman $\mathrm{H}$, et al. Effect of physical exercise on cognitive performance in older adults with mild cognitive impairment or dementia: A systematic review. Dement Geriatr Cogn Disord. 2014;38(5-6):347-65.

[8] Barha CK, et al. Sex differences in exercise efficacy to improve cognition: A systematic review and meta-analysis of randomized controlled trials in older humans. Front Neuroendocrinol. 2017;46:71-85.

[9] Podcasy JL, Epperson CN. Considering sex and gender in Alzheimer disease and other dementias. Dialogues Clin Neurosci. 2016;18(4):437-46.

[10] Lin KA, Doraiswamy PM. When mars versus venus is not a cliche: Gender differences in the neurobiology of Alzheimer's disease. Front Neurol. 2014;5:288.

[11] Ardekani BA, Convit A, Bachman AH. Analysis of the MIRIAD data shows sex differences in hippocampal atrophy progression. J Alzheimers Dis. 2016;50(3): 847-57.

[12] Hua X, et al. Sex and age differences in atrophic rates: An ADNI study with $n=1368$ MRI scans. Neurobiol Aging. 2010;31(8):1463-80.

[13] Gao S, et al. The relationships between age, sex, and the incidence of dementia and Alzheimer disease: A metaanalysis. Arch Gen Psychiatry. 1998;55(9):809-15.

[14] Jakubs K, et al. Inflammation regulates functional integration of neurons born in adult brain. J Neurosci. 2008;28(47):12477-88.

[15] Barnes LL, et al. Sex differences in the clinical manifestations of Alzheimer disease pathology. Arch Gen Psychiatry. 2005;62(6):685-91.

[16] Petersen RC. Mild cognitive impairment as a diagnostic entity. J Intern Med. 2004;256(3):183-94.

[17] Roberts RO, et al. The incidence of MCI differs by subtype and is higher in men: The Mayo Clinic Study of Aging. Neurology. 2012;78(5):342-51.

[18] Caracciolo B, et al. Occurrence of cognitive impairment and dementia in the community: A 9-year-long prospective study. Neurology. 2008;70(19 Pt 2):1778-85.

[19] Brodaty $\mathrm{H}$, et al. Mild cognitive impairment in a community sample: The Sydney Memory and Ageing Study. Alzheimers Dement. 2013;9(3):310-317 e1.

[20] Katz MJ, et al. Age-specific and sex-specific prevalence and incidence of mild cognitive impairment, dementia, and
Alzheimer dementia in blacks and whites: A report from the Einstein Aging Study. Alzheimer Dis Assoc Disord. 2012;26(4):335-43.

[21] Fitzpatrick AL, et al. Incidence and prevalence of dementia in the Cardiovascular Health Study. J Am Geriatr Soc. 2004;52(2):195-204.

[22] Sundermann EE, et al. Female advantage in verbal memory: Evidence of sex-specific cognitive reserve. Neurology. 2016;87(18):1916-24.

[23] Sundermann EE, et al. Better verbal memory in women than men in MCI despite similar levels of hippocampal atrophy. Neurology. 2016;86(15):1368-76.

[24] Brouwers N, Sleegers K, Van Broeckhoven C. Molecular genetics of Alzheimer's disease: An update. Ann Med. 2008;40(8):562-83.

[25] Farrer LA, et al. Effects of age, sex, and ethnicity on the association between apolipoprotein E genotype and Alzheimer disease. A meta-analysis. APOE and Alzheimer Disease Meta Analysis Consortium. JAMA. 1997;278(16):1349-56.

[26] Neu SC, et al. Apolipoprotein E genotype and sex risk factors for Alzheimer Disease: A meta-analysis. JAMA Neurol. 2017;74(10):1178-89.

[27] Kim S, et al. Gender differences in risk factors for transition from mild cognitive impairment to Alzheimer's disease: A CREDOS study. Compr Psychiatry. 2015;62:114-22.

[28] Altmann A, et al. Sex modifies the APOE-related risk of developing Alzheimer disease. Ann Neurol. 2014;75(4):563-73.

[29] Corder EH, et al. The biphasic relationship between regional brain senile plaque and neurofibrillary tangle distributions: Modification by age, sex, and APOE polymorphism. Ann N Y Acad Sci. 2004;1019:24-8.

[30] Beydoun MA, et al. Sex differences in the association of the apolipoprotein E epsilon 4 allele with incidence of dementia, cognitive impairment, and decline. Neurobiol Aging. 2012;33(4):720-731 e4.

[31] Alvarez A, et al. Apathy and APOE4 are associated with reduced BDNF levels in Alzheimer's disease. J Alzheimers Dis. 2014;42(4):1347-55.

[32] Cacciottolo M, et al. The APOE4 allele shows opposite sex bias in microbleeds and Alzheimer's disease of humans and mice. Neurobiol Aging. 2016;37:47-57.

[33] Dufouil C, Seshadri S, Chene G. Cardiovascular risk profile in women and dementia. J Alzheimers Dis. 2014;42(Suppl 4):S353-63.

[34] West RK, et al. Waist circumference is correlated with poorer cognition in elderly type 2 diabetes women. Alzheimers Dement. 2016;12(8):925-9.

[35] Pankratz VS, et al. Predicting the risk of mild cognitive impairment in the Mayo Clinic Study of Aging. Neurology. 2015;84(14):1433-42.

[36] Chatterjee $S$, et al. Type 2 diabetes as a risk factor for dementia in women compared with men: A pooled analysis of 2.3 million people comprising more than 100,000 cases of dementia. Diabetes Care. 2016;39(2):300-7.

[37] Hall JR, et al. Biomarkers of vascular risk, systemic inflammation, and microvascular pathology and neuropsychiatric symptoms in Alzheimer's disease. J Alzheimers Dis. 2013;35(2):363-71.

[38] Taddei S, et al. Menopause is associated with endothelial dysfunction in women. Hypertension. 1996;28(4): 576-82. 
[39] Trollor JN, et al. The association between systemic inflammation and cognitive performance in the elderly: The Sydney Memory and Ageing Study. Age (Dordr). 2012;34(5):1295-308.

[40] Ozturk C, et al. The diagnostic role of serum inflammatory and soluble proteins on dementia subtypes: Correlation with cognitive and functional decline. Behav Neurol. 2007;18(4):207-15.

[41] Canon ME, Crimmins EM. Sex differences in the association between muscle quality, inflammatory markers, and cognitive decline. J Nutr Health Aging. 2011;15(8):695-8.

[42] Berchtold NC, et al. Gene expression changes in the course of normal brain aging are sexually dimorphic. Proc Natl Acad Sci U S A. 2008;105(40):15605-10.

[43] Mangold CA, et al. Sexually divergent induction of microglial-associated neuroinflammation with hippocampal aging. J Neuroinflammation. 2017;14(1):141.

[44] Mouton PR, et al. Age and gender effects on microglia and astrocyte numbers in brains of mice. Brain Res. 2002;956(1):30-5

[45] Hanamsagar R, et al. Generation of a microglial developmental index in mice and in humans reveals a sex difference in maturation and immune reactivity. Glia. 2017;65(9):1504-20.

[46] Ferretti MT, Cuello AC. Does a pro-inflammatory process precede Alzheimer's disease and mild cognitive impairment? Curr Alzheimer Res. 2011;8(2):164-74.

[47] Feldman HA, et al. Age trends in the level of serum testosterone and other hormones in middle-aged men: Longitudinal results from the Massachusetts male aging study. J Clin Endocrinol Metab. 2002;87(2):589-98.

[48] Decaroli MC, Rochira V. Aging and sex hormones in males. Virulence. 2017;8(5):545-70.

[49] Mosconi L, et al. Sex differences in Alzheimer risk: Brain imaging of endocrine vs chronologic aging. Neurology. 2017;89(13):1382-90

[50] Rocca WA, et al. Increased risk of cognitive impairment or dementia in women who underwent oophorectomy before menopause. Neurology. 2007;69(11):1074-83.

[51] Rocca WA, Grossardt BR, Shuster LT. Oophorectomy, menopause, estrogen treatment, and cognitive aging: Clinical evidence for a window of opportunity. Brain Res. 2011;1379:188-98.

[52] Brinton RD. The healthy cell bias of estrogen action: Mitochondrial bioenergetics and neurological implications. Trends Neurosci. 2008;31(10):529-37.

[53] Barha CK, Dalton GL, Galea LA. Low doses of 17alphaestradiol and 17beta-estradiol facilitate, whereas higher doses of estrone and 17alpha- and 17beta-estradiol impair, contextual fear conditioning in adult female rats. Neuropsychopharmacology. 2010;35(2):547-59.

[54] Barha CK, Galea LA. The hormone therapy, Premarin, impairs hippocampus-dependent spatial learning and memory and reduces activation of new granule neurons in response to memory in female rats. Neurobiol Aging. 2013;34(3):986-1004.

[55] Barha CK, Galea LA. Influence of different estrogens on neuroplasticity and cognition in the hippocampus. Biochim Biophys Acta. 2010;1800(10):1056-67.

[56] Stern Y, et al. Influence of education and occupation on the incidence of Alzheimer's disease. JAMA. 1994;271(13):1004-10.

[57] Stern Y, et al. Rate of memory decline in $\mathrm{AD}$ is related to education and occupation: Cognitive reserve? Neurology. 1999;53(9):1942-7.
[58] Le Carret N, et al. Influence of education on the pattern of cognitive deterioration in $\mathrm{AD}$ patients: The cognitive reserve hypothesis. Brain Cogn. 2005;57(2):120-6.

[59] Hall CB, et al. Education delays accelerated decline on a memory test in persons who develop dementia. Neurology. 2007;69(17):1657-64.

[60] Mielke MM, Vemuri P, Rocca WA. Clinical epidemiology of Alzheimer's disease: Assessing sex and gender differences. Clin Epidemiol. 2014;6:37-48.

[61] Winblad B, et al. Defeating Alzheimer's disease and other dementias: A priority for European science and society. Lancet Neurol. 2016;15(5):455-532.

[62] Epel ES, et al. Accelerated telomere shortening in response to life stress. Proc Natl Acad Sci U S A. 2004;101(49):17312-5.

[63] Norton MC, et al. Greater risk of dementia when spouse has dementia? The Cache County study. J Am Geriatr Soc. 2010;58(5):895-900.

[64] Cotman CW, Berchtold NC, Christie LA. Exercise builds brain health: Key roles of growth factor cascades and inflammation. Trends Neurosci. 2007;30(9):464-72.

[65] Prakash RS, et al. Physical activity and cognitive vitality. Annu Rev Psychol. 2015;66:769-97.

[66] Voss MW, et al. Bridging animal and human models of exercise-induced brain plasticity. Trends Cogn Sci. 2013; 17(10):525-44.

[67] Northey JM, et al. Exercise interventions for cognitive function in adults older than 50: A systematic review with meta-analysis. Br J Sports Med. 2018;52(3):154-60.

[68] Barnes DE, et al. A longitudinal study of cardiorespiratory fitness and cognitive function in healthy older adults. J Am Geriatr Soc. 2003;51(4):459-65.

[69] Zhu W, et al. Objectively measured physical activity and cognitive function in older adults. Med Sci Sports Exerc. 2017;49(1):47-53.

[70] Stubbs B, et al. Accelerometer-assessed light physical activity is protective of future cognitive ability: A longitudinal study among community dwelling older adults. Exp Gerontol. 2017;91:104-9.

[71] Olaya B, et al. Trajectories of verbal episodic memory in middle-aged and older adults: Evidence from the English longitudinal study of ageing. J Am Geriatr Soc. 2017;65(6):1274-81.

[72] Heyn P, Abreu BC, Ottenbacher KJ. The effects of exercise training on elderly persons with cognitive impairment and dementia: A meta-analysis. Arch Phys Med Rehabil. 2004;85(10):1694-704.

[73] Etnier JL, et al. A meta-regression to examine the relationship between aerobic fitness and cognitive performance. Brain Res Rev. 2006;52(1):119-30.

[74] Smith PJ, et al. Aerobic exercise and neurocognitive performance: A meta-analytic review of randomized controlled trials. Psychosom Med. 2010;72(3):239-52.

[75] Kelly ME, et al. The impact of exercise on the cognitive functioning of healthy older adults: A systematic review and meta-analysis. Ageing Res Rev. 2014;16:12-31.

[76] Young J, et al. Aerobic exercise to improve cognitive function in older people without known cognitive impairment. Cochrane Database Syst Rev. 2015(4):CD005381.

[77] Baker LD, et al. Effects of aerobic exercise on mild cognitive impairment: A controlled trial. Arch Neurol. 2010;67(1):71-9.

[78] Liu-Ambrose T, et al. Aerobic exercise and vascular cognitive impairment: A randomized controlled trial. Neurology. 2016;87(20):2082-90. 
[79] Erickson KI, et al. Exercise training increases size of hippocampus and improves memory. Proc Natl Acad Sci U S A. $2011 ; 108(7): 3017-22$.

[80] Nagamatsu LS, et al. Physical activity improves verbal and spatial memory in older adults with probable mild cognitive impairment: A 6-month randomized controlled trial. J Aging Res. 2013;2013:861893.

[81] Kramer AF, et al. Ageing, fitness and neurocognitive function. Nature. 1999;400(6743):418-9.

[82] Gajewski PD, Falkenstein M. Physical activity and neurocognitive functioning in aging - a condensed updated review. Eur Rev Aging Phys Act. 2016;13:1.

[83] Nishiguchi S, et al. A 12-week physical and cognitive exercise program can improve cognitive function and neural efficiency in community-dwelling older adults: A randomized controlled trial. J Am Geriatr Soc. 2015;63(7):1355-63.

[84] Voss MW, et al. Plasticity of brain networks in a randomized intervention trial of exercise training in older adults. Front Aging Neurosci. 2010;2.

[85] ten Brinke LF, et al. Aerobic exercise increases hippocampal volume in older women with probable mild cognitive impairment: A 6-month randomised controlled trial. Br J Sports Med. 2015;49(4):248-54.

[86] Hsu CL, et al. The impact of aerobic exercise on frontoparietal network connectivity and its relation to mobility: An exploratory analysis of a 6-month randomized controlled trial. Front Hum Neurosci. 2017;11:344.

[87] Hsu CL, et al. Aerobic exercise promotes executive functions and impacts functional neural activity among older adults with vascular cognitive impairment. Br J Sports Med. 2017.

[88] Baniqued PL, et al. Brain network modularity predicts exercise-related executive function gains in older adults. Front Aging Neurosci. 2017;9:426.

[89] Liu-Ambrose T, et al. Resistance training and executive functions: A 12-month randomized controlled trial. Arch Intern Med. 2010;170(2):170-8.

[90] Best JR, et al. Long-term effects of resistance exercise training on cognition and brain volume in older women: Results from a randomized controlled trial. J Int Neuropsychol Soc. 2015;21(10):745-56.

[91] Cassilhas RC, et al. The impact of resistance exercise on the cognitive function of the elderly. Med Sci Sports Exerc. 2007;39(8):1401-7.

[92] Liu-Ambrose T, et al. Otago home-based strength and balance retraining improves executive functioning in older fallers: A randomized controlled trial. J Am Geriatr Soc. 2008;56(10):1821-30.

[93] Nagamatsu LS, et al. Resistance training promotes cognitive and functional brain plasticity in seniors with probable mild cognitive impairment. Arch Intern Med. 2012;172(8):666-8.

[94] Liu-Ambrose T, et al. Resistance training and functional plasticity of the aging brain: A 12-month randomized controlled trial. Neurobiol Aging. 2012;33(8):1690-8.

[95] Bolandzadeh N, et al. Resistance training and white matter lesion progression in older women: Exploratory analysis of a 12-month randomized controlled trial. J Am Geriatr Soc. 2015;63(10):2052-60.

[96] Wanderley FA, et al. Differential responses of adiposity, inflammation and autonomic function to aerobic versus resistance training in older adults. Exp Gerontol. 2013;48(3):326-33.
[97] Cassilhas RC, et al. Spatial memory is improved by aerobic and resistance exercise through divergent molecular mechanisms. Neuroscience. 2012;202:309-17.

[98] Cornelissen VA, Fagard RH. Effect of resistance training on resting blood pressure: A meta-analysis of randomized controlled trials. J Hypertens. 2005;23(2):251-9.

[99] Cotman CW, Berchtold NC. Exercise: A behavioral intervention to enhance brain health and plasticity. Trends Neurosci. 2002;25(6):295-301.

[100] Dishman RK, et al. Neurobiology of exercise. Obesity (Silver Spring). 2006;14(3):345-56.

[101] Williams MA, et al. Resistance exercise in individuals with and without cardiovascular disease: 2007 update: A scientific statement from the American Heart Association Council on Clinical Cardiology and Council on Nutrition, Physical Activity, and Metabolism. Circulation. 2007;116(5):572-84.

[102] Strasser B, Siebert U, Schobersberger W. Resistance training in the treatment of the metabolic syndrome: A systematic review and meta-analysis of the effect of resistance training on metabolic clustering in patients with abnormal glucose metabolism. Sports Med. 2010;40(5): 397-415.

[103] Taaffe DR, et al. Reduced central blood pressure in older adults following progressive resistance training. J Hum Hypertens. 2007;21(1):96-8.

[104] Donges CE, Duffield R, Drinkwater EJ. Effects of resistance or aerobic exercise training on interleukin-6, C-reactive protein, and body composition. Med Sci Sports Exerc. 2010;42(2):304-13.

[105] Mattusch F, et al. Reduction of the plasma concentration of C-reactive protein following nine months of endurance training. Int J Sports Med. 2000;21(1):21-4.

[106] Kaplan MS, et al. Demographic and psychosocial correlates of physical activity in late life. Am J Prev Med. 2001;21(4):306-12.

[107] Lee YS. Gender differences in physical activity and walking among older adults. J Women Aging. 2005;17(12):55-70.

[108] Fagot D, et al. The impact of physical activity and sex differences on intraindividual variability in inhibitory performance in older adults. Neuropsychol Dev Cogn B Aging Neuropsychol Cogn. 2017;Sep 4:1-23.

[109] Barha CK, et al. Sex differences in aerobic exercise efficacy to improve cognition: A systematic review and meta-analysis of studies in older rodents. Front Neuroendocrinol. 2017;46:86-105.

[110] Barha CK, et al. Sex difference in aerobic exercise efficacy to improve cognition in older adults with vascular cognitive impairment: Secondary analysis of a randomized controlled trial. J Alzheimers Dis. 2017;60(4):1397-410.

[111] van Uffelen JG, et al. Walking or vitamin B for cognition in older adults with mild cognitive impairment? A randomised controlled trial. Br J Sports Med. 2008;42(5):344-51.

[112] Varma VR, et al. Low-intensity daily walking activity is associated with hippocampal volume in older adults. Hippocampus. 2015;25(5):605-15.

[113] Varma VR, Tang X, Carlson MC. Hippocampal subregional shape and physical activity in older adults. Hippocampus. 2016;26(8):1051-60.

[114] Trivino-Paredes J, et al. The effects of hormones and physical exercise on hippocampal structural plasticity. Front Neuroendocrinol. 2016;41:23-43. 
[115] Voss MW, et al. Exercise, brain, and cognition across the life span. J Appl Physiol (1985). 2011;111(5):1505-13.

[116] Szuhany KL, M. Bugatti, Otto MW. A meta-analytic review of the effects of exercise on brain-derived neurotrophic factor. J Psychiatr Res. 2015;60:56-64.

[117] Huang T, et al. The effects of physical activity and exercise on brain-derived neurotrophic factor in healthy humans: A review. Scand J Med Sci Sports. 2014;24(1):1-10.

[118] Coelho FG, et al. Physical exercise modulates peripheral levels of brain-derived neurotrophic factor (BDNF): A systematic review of experimental studies in the elderly. Arch Gerontol Geriatr. 2013;56(1):10-5.

[119] Knaepen K, et al. Neuroplasticity - exercise-induced response of peripheral brain-derived neurotrophic factor: A systematic review of experimental studies in human subjects. Sports Med. 2010;40(9):765-801.

[120] Phillips C, et al. Neuroprotective effects of physical activity on the brain: A closer look at trophic factor signaling. Front Cell Neurosci. 2014;8:170.

[121] Atwi S, et al. Androgen modulation of hippocampal structure and function. Neuroscientist. 2016;22(1):46-60.

[122] Leal G, et al. Regulation of hippocampal synaptic plasticity by BDNF. Brain Res. 2015;1621:82-101.

[123] Chan CB, Ye K. Sex differences in brain-derived neurotrophic factor signaling and functions. J Neurosci Res. 2017;95(1-2):328-35.

[124] Scharfman HE, MacLusky NJ. Sex differences in hippocampal area CA3 pyramidal cells. J Neurosci Res. 2017;95(1-2):563-75.

[125] Trivino-Paredes J, et al. The effects of hormones and physical exercise on hippocampal structural plasticity. Front Neuroendocrinol. 2016.

[126] Dachtler J, Fox K. Do cortical plasticity mechanisms differ between males and females? J Neurosci Res. 2017;95(12):518-26.

[127] Choleris E, et al. Sex differences in the brain: Implications for behavioral and biomedical research. Neurosci Biobehav Rev. 2018;85:126-45.

[128] Woolley CS, McEwen BS. Roles of estradiol and progesterone in regulation of hippocampal dendritic spine density during the estrous cycle in the rat. J Comp Neurol. 1993;336(2):293-306.

[129] Leranth C, Petnehazy O, MacLusky NJ. Gonadal hormones affect spine synaptic density in the CA1 hippocampal subfield of male rats. J Neurosci. 2003;23(5): 1588-92.

[130] Leranth C, Hajszan T, MacLusky NJ. Androgens increase spine synapse density in the CA1 hippocampal subfield of ovariectomized female rats. J Neurosci. 2004;24(2):495-9.

[131] Galea LA, et al. Sex differences in dendritic atrophy of CA3 pyramidal neurons in response to chronic restraint stress. Neuroscience. 1997;81(3):689-97.

[132] McEwen BS, Morrison JH. The brain on stress: Vulnerability and plasticity of the prefrontal cortex over the life course. Neuron. 2013;79(1):16-29.

[133] Pawluski JL, et al. Effects of steroid hormones on neurogenesis in the hippocampus of the adult female rodent during the estrous cycle, pregnancy, lactation and aging. Front Neuroendocrinol. 2009;30(3):343-57.

[134] Chow C, et al. Sex differences in neurogenesis and activation of new neurons in response to spatial learning and memory. Psychoneuroendocrinology. 2013;38(8): 1236-50.

[135] Dalla C, et al. Female rats learn trace memories better than male rats and consequently retain a greater proportion of new neurons in their hippocampi. Proc Natl Acad Sci U S A. 2009;106(8):2927-32.

[136] Barha CK, et al. Chronic restraint stress in adolescence differentially influences hypothalamic-pituitary-adrenal axis function and adult hippocampal neurogenesis in male and female rats. Hippocampus. 2011;21(11):1216-27.

[137] Barker JM, Galea LA. Repeated estradiol administration alters different aspects of neurogenesis and cell death in the hippocampus of female, but not male, rats. Neuroscience. 2008;152(4):888-902.

[138] Sohrabji F, Miranda RC, Toran-Allerand CD. Identification of a putative estrogen response element in the gene encoding brain-derived neurotrophic factor. Proc Natl Acad Sci U S A. 1995;92(24):11110-4.

[139] Harte-Hargrove LC, Maclusky NJ, Scharfman HE. Brain-derived neurotrophic factor-estrogen interactions in the hippocampal mossy fiber pathway: Implications for normal brain function and disease. Neuroscience. 2013;239:46-66.

[140] Scharfman HE, MacLusky NJ. Differential regulation of BDNF, synaptic plasticity and sprouting in the hippocampal mossy fiber pathway of male and female rats. Neuropharmacology. 2014;76(Pt C):696-708.

[141] Komulainen P, et al. BDNF is a novel marker of cognitive function in ageing women: The DR's EXTRA Study. Neurobiol Learn Mem. 2008;90(4):596-603.

[142] Egan MF, et al. The BDNF val66met polymorphism affects activity-dependent secretion of BDNF and human memory and hippocampal function. Cell. 2003;112(2):257-69.

[143] Fukumoto N, et al. Sexually dimorphic effect of the Val66Met polymorphism of BDNF on susceptibility to Alzheimer's disease: New data and meta-analysis. Am J Med Genet B Neuropsychiatr Genet. 2010;153B(1): $235-42$.

[144] Laing KR, et al. Brain-derived neurotrophic factor (BDNF) gene: A gender-specific role in cognitive function during normal cognitive aging of the MEMO-Study? Age (Dordr). 2012;34(4):1011-22.

[145] Nemoto K, et al. The Val66Met polymorphism of the brain-derived neurotrophic factor gene affects age-related brain morphology. Neurosci Lett. 2006;397(1-2):25-9.

[146] Wei SM, et al. Brain-derived neurotrophic factor Val(6)(6)Met polymorphism affects resting regional cerebral blood flow and functional connectivity differentially in women versus men. J Neurosci. 2012;32(20):7074-81.

[147] Spencer JL, et al. BDNF variant Val66Met interacts with estrous cycle in the control of hippocampal function. Proc Natl Acad Sci U S A. 2010;107(9):4395-400.

[148] Harms CA, Rosenkranz S. Sex differences in pulmonary function during exercise. Med Sci Sports Exerc. 2008;40(4):664-8.

[149] Sheel AW, et al. Sex differences in respiratory exercise physiology. Sports Med. 2004;34(9):567-79.

[150] Weiss EP, et al. Gender differences in the decline in aerobic capacity and its physiological determinants during the later decades of life. J Appl Physiol (1985). 2006;101(3): 938-44.

[151] Stathokostas L, et al. Longitudinal changes in aerobic power in older men and women. J Appl Physiol (1985). 2004;97(2):781-9.

[152] Tarnopolsky MA, Sex differences in exercise metabolism and the role of 17-beta estradiol. Med Sci Sports Exerc. 2008;40(4):648-54.

[153] Goodpaster BH, et al. The loss of skeletal muscle strength, mass, and quality in older adults: The health, aging and 
body composition study. J Gerontol A Biol Sci Med Sci. 2006;61(10):1059-64.

[154] Borde R, Hortobagyi T, Granacher U. Dose-response relationships of resistance training in healthy old adults: A systematic review and meta-analysis. Sports Med. 2015;45(12):1693-720.

[155] Da Boit M, et al. Sex differences in the response to resistance exercise training in older people. Physiol Rep. 2016;4(12).

[156] Leenders M, et al. Elderly men and women benefit equally from prolonged resistance-type exercise training. J Gerontol A Biol Sci Med Sci. 2013;68(7):769-79.

[157] Da Boit M, et al. Sex differences in the effect of fishoil supplementation on the adaptive response to resistance exercise training in older people: A randomized controlled trial. Am J Clin Nutr. 2017;105(1):151-8.

[158] Merz AA, Cheng S. Sex differences in cardiovascular ageing. Heart. 2016;102(11):825-31.

[159] Goldspink DF, et al. A study of presbycardia, with gender differences favoring ageing women. Int $\mathrm{J}$ Cardiol. 2009;137(3):236-45.
[160] Martin WH 3rd, et al. Effects of aging, gender, and physical training on peripheral vascular function. Circulation. 1991;84(2):654-64.

[161] Martin WH 3rd, et al. Exercise training enhances leg vasodilatory capacity of 65-yr-old men and women. J Appl Physiol (1985). 1990;69(5):1804-9.

[162] DeSouza CA, et al. Regular aerobic exercise prevents and restores age-related declines in endotheliumdependent vasodilation in healthy men. Circulation. 2000;102(12):1351-7.

[163] Pierce GL, et al. Sex-specific effects of habitual aerobic exercise on brachial artery flow-mediated dilation in middle-aged and older adults. Clin Sci (Lond). 2011;120(1):13-23.

[164] Moreau KL, et al. Essential role of estrogen for improvements in vascular endothelial function with endurance exercise in postmenopausal women. J Clin Endocrinol Metab. 2013;98(11):4507-15. 